\title{
Ultrasound microbubble contrast agent-mediated suicide gene transfection in the treatment of hepatic cancer
}

\author{
QING TANG $^{1}$, XIAOHONG HE ${ }^{2}$, HAIXING LIAO ${ }^{1}$, LIANTU HE ${ }^{1}$, YING WANG ${ }^{1}$, \\ DAZHI ZHOU ${ }^{1}$, SUMIN YE ${ }^{1}$ and QILU CHEN ${ }^{1}$ \\ ${ }^{1}$ Department of Sonography, The First Affiliated Hospital of Guangzhou Medical College, \\ Guangzhou, Guangdong 510120; ${ }^{2}$ Department of Laboratory Medicine, The First Affiliated \\ Hospital of Sun Yat-sen University, Guangzhou, Guangdong 510080, P.R. China
}

Received April 10, 2012; Accepted July 17, 2012

DOI: $10.3892 / \mathrm{ol} .2012 .845$

\begin{abstract}
The aim of this study was to investigate the role of ultrasound microbubble contrast agent-mediated suicide gene transfection in the treatment of hepatic cancer. We intratumorally injected KDR-TK, AFP-TK and microbubble contrast agent into nude mice prior to ultrasound treatment and administration of prodrugs (GCV and 5-FC). The tumor volume, tumor inhibition rate, survival time and apoptosis of tumor cells was determined. The sizes of subcutaneous hepatic cancers in mice receiving treatment were comparable to those in the control group, and the survival time was similar between the two groups $(\mathrm{P}>0.05)$. However, the tumor inhibition rate and the number of apoptotic cells in the treatment group was markedly higher compared with that in the control group $(\mathrm{P}<0.05)$. Evident tumor necrosis was absent in both groups, except at the needle tract. Ultrasound therapy following injection of suicide genes and microbubble contrast agents is able to inhibit cancer growth in vivo. This may be attributed to the induction of cancer cell apoptosis.
\end{abstract}

\section{Introduction}

Herpes simplex virus-thymidine kinase/ganciclovir (HSV-TK/GCV) is the most widely used suicide gene/prodrug system. During gene therapy, the target gene is transfected into target cells. However, there are no safe or effective strategies for gene transfection in clinical practice, which significantly limits the application of gene therapy. Although gene transfection via viral vectors has been demonstrated to have a high transfection efficiency, viral vectors have a risk of inducing an immune response or other toxic reactions in the host $(1,2)$. Ultrasound has been applied in gene therapy and in recent years, an ultrasound contrast agent has been identified as an adjuvant to increase

Correspondence to: Dr Xiaohong He, Department of Laboratory Medicine, The First Affiliated Hospital of Sun Yat-sen University, 151 West Yanjiang Road, Guangzhou, Guangdong 510080, P.R. China E-mail: he810218@sina.com

Key words: gene therapy, hepatic cancer, ultrasound cavitation effect, microbubble contrast agent the efficiency of ultrasound-mediated gene transfection. The application of ultrasound treatment and an ultrasound contrast agent for gene transfection is a more favorable strategy for gene therapy, as it has a high safety potential and the ability to directly target cells $(3,4)$. In this study, we constructed eukaryotic expression vectors expressing the thymidine kinase (TK) gene, which contained either the AFP promoter (specific in hepatic cancer cells) or the kinase insert domain receptor (KDR) promoter (specific in vascular endothelial cells). In combination with a microbubble contrast agent, the two vectors were intratumorally injected into mice, ultrasound treatment was conducted and prodrugs [GCV and 5-fluorocy tosine (5-FC)] were administered. The aim of this study was to investigate the specific therapeutic effect of HSV-TK/GCV and cytosine deaminase (CD)/5-FC on hepatic cancer in vivo.

\section{Materials and methods}

Animals and cell lines. Male C57BL/6 nude mice approximately 4-5 weeks old and weighing 17-19 g were purchased from the Experimental Animal Center of Sun Yat-sen University (Guangdong, China). The human hepatic cancer cell line (HepG2) was kindly provided by the Surgical Laboratory of The First Affiliated Hospital of Sun Yat-sen University. The study was approved by the First Affiliated Hospital of Sun Yat-sen University

Materials and instruments. Transcranial Doppler ultrasound (output sound intensity, $0-3 \mathrm{~W} / \mathrm{cm}^{2}$; radiation frequency, $1 \mathrm{MHz}$; duty cycle, 10-100\%) and a plasmid extraction purification kit were obtained from Qiagen (Valencia, CA, USA). Double Stain Apoptosis Detection kit (Hoechst 33342/PI) was purchased from Byeotime Institution of Biotechnology (Jiangsu, China) and pEGFP-KDR-TK and pEGFP-C1-AFP-TK were provided by Dr Li JB from Shenzhen Hospital of Peiking University (Beijing, China). SonoVue ultrasound contrast $(59 \mathrm{mg} /$ bottle of white freeze-dried powder in sulfur hexafluoride with $2.5 \mu \mathrm{m}$ microbubble) was purchased from Bracco Imaging BV (Milan, Italy). The fluorescence microscope, freezing microtome and GCV were purchased from Lizhu Keyi Pharmaceuticals Co., Ltd (Wuhan, China) and 5-FC was purchased from Sigma (St. Louis, MO, USA). SonoVue solu- 
Table I. Tumor volume and inhibition rate in cancer-bearing mice following treatment.

Tumor volume $\left(\mathrm{mm}^{3}\right)$

\begin{tabular}{|c|c|c|c|c|c|}
\hline \multirow[b]{2}{*}{ Group } & & \multirow{2}{*}{$\begin{array}{c}\text { Inhibition } \\
\text { rate }(\%)\end{array}$} \\
\hline & Day 3 & Day 6 & Day 9 & Day 12 & \\
\hline A & $875.8 \pm 27.4$ & $1087.5 \pm 30.1$ & $1187.1 \pm 45.8$ & $1451.2 \pm 48.0$ & 0 \\
\hline B & $886.6 \pm 29.5$ & $971.9 \pm 41.6$ & $1140.5 \pm 42.2$ & $1396.5 \pm 46.8$ & $3.5 \pm 1.8$ \\
\hline $\mathrm{C}$ & $847.8 \pm 24.1$ & $969.4 \pm 59.3$ & $1035.1 \pm 66.0$ & $1205.6 \pm 47.1$ & $9.7 \pm 3.7^{\mathrm{a}}$ \\
\hline D & $834.9 \pm 25.2$ & $953.6 \pm 70.5$ & $987.4 \pm 85.9$ & $1127.3 \pm 63.8$ & $10.6 \pm 4.9^{a}$ \\
\hline
\end{tabular}

${ }^{\mathrm{a}} \mathrm{P}<0.05$ vs. groups $\mathrm{A}$ and $\mathrm{B}$.

tion was prepared by gently agitating dissolved SonoVue in $5 \%$ PBS.

Preparation of subcutaneous hepatic cancer model in nude mice. A total of 24 mice were housed in an aseptic environment. At week 7, HepG2 cells $\left(0.2 \mathrm{ml} ; 1 \times 10^{7}\right.$ cells) were subcutaneously injected into the lateral part of the back. After 1 week, the subcutaneous nodules were identified. When the tumor volume reached $100 \mathrm{~mm}^{3}$, animals were selected for the following experiments. The drugs were mixed in a 1-ml syringe and injected into the upper outer, lower outer, lower inner and upper inner quadrants of the tumor. The tumor was then palpated and ultrasound treatment was conducted. The probe was placed on the tumor and close to the skin, and the couplant was positioned between the probe and the skin. The probe frequency was $1 \mathrm{MHz}$, the sound intensity was $2 \mathrm{~W} / \mathrm{cm}^{2}$, the time for ultrasound treatment was $2 \mathrm{~min}$ and the duty cycle was $50 \%$.

Grouping. The nude mice were randomly assigned into 1 of 4 groups ( $n=6$ per group). Group A (control group) were administered $100 \mu \mathrm{l}$ of normal saline. Group B were administered $100 \mu \mathrm{l}$ of normal saline, $50 \mu \mathrm{g}$ of pEGFP-KDR-TK and $50 \mu \mathrm{g}$ of pEGFP-C1-AFP-TK. Group $\mathrm{C}$ were administered $100 \mu \mathrm{l}$ of normal saline, $50 \mu \mathrm{g}$ of pEGFP-KDR-TK and $50 \mu \mathrm{g}$ of pEGFP-C1-AFP-TK, and ultrasound was conducted. Group D were administered $100 \mu \mathrm{l}$ of normal saline, $50 \mu \mathrm{g}$ of pEGFP-KDR-TK, $50 \mu \mathrm{g}$ pEGFP-C1-AFP-TK and 5\% SonoVue, and ultrasound was conducted.

Intratumoral injection and ultrasound treatment were conducted once a day for 3 consecutive days. Subsequently, the mice received an intraperitoneal injection of GCV (40 mg/kg/day) and 5-FC (40 mg/kg/day) for 10 consecutive days. The tumor volume, tumor inhibition rate and apoptosis of cancer cells (using Hoechst staining) were determined.

Observations. The tumor volume was measured regularly. The vernier caliper was used to measure the maximal and minimal diameter of the tumor $(\mathrm{mm})$ which were expressed as a and b, respectively. The tumor volume was calculated as: $\mathrm{V} \mathrm{mm} \mathrm{m}^{3}=1 / 2 \times \mathrm{a} \mathrm{x}^{2}$. Measurements were conducted once every 3 days, and were collected a total of 4 times. The tumor growth curve was delineated and the tumor growth inhibition rate was calculated: Inhibition rate $=$ (mean tumor volume control - mean tumor volume treatment)/mean tumor volume control x 100. After 12 days, mice were sacrificed $(n=3$ per group) and the tumors were collected and stored in liquid nitrogen. The survival times of the 3 remaining cancer-bearing mice were also recorded and analyzed for 90 consecutive days.

Hoechst and hematoxylin and eosin $(H \& E)$ staining. Frozen sections were obtained and observed under a fluorescence microscope (excitation wavelength, $350 \mathrm{~nm}$; emission wavelength, $460 \mathrm{~nm}$ ) and cells with a blue nuclei were presented. A total of 3 fields were randomly selected (magnification, x400), and the total number of cells and the number of apoptotic cells were counted. The proportion of apoptotic cells in all cells was calculated: Apoptosis index $(\mathrm{AI})=$ number of apoptotic cells/number of total cells $\mathrm{x} 100$. The sections undergoing H\&E staining were observed under a light microscope.

Statistical analysis. SPSS version 13.0 was used for statistical analysis, and qualitative data were expressed as the mean \pm standard deviation. A one way analysis of variance was employed for comparisons among groups and Fisher's Least Significant Difference (LSD) test was conducted for comparisons between two groups. $\mathrm{P}<0.05$ was considered to indicate a statistically significant difference. Survival analysis was conducted using the Kaplan-Meier method.

\section{Results}

Tumor growth. The tumor volume and inhibition rate 3, 6, 9 and 12 days after treatment are shown in Table I. Statistical analysis revealed that no marked difference was observed in the tumor inhibition rate and tumor volume between any two groups $(\mathrm{P}>0.05)$. However, the tumor inhibition rate in groups $\mathrm{D}$ and $\mathrm{C}$ was markedly different compared with that in groups $\mathrm{A}$ and $\mathrm{B}(\mathrm{P}<0.05)$.

Survival time of cancer-bearing mice. After treatment, the survival times were $46.33 \pm 19,45.68 \pm 24,50.27 \pm 11$ and $48.40 \pm 17$ days in groups A, B, C and D, respectively. No marked difference was identified in the survival time between the two groups $(\mathrm{P}>0.05)$.

Apoptosis of cancer cells. Compared with groups A and B, the number of apoptotic cells in groups $\mathrm{C}$ and $\mathrm{D}$ was markedly higher. The AI was $3 \pm 1.1,8 \pm 2.6,17 \pm 3.4$ and $19 \pm 5.5 \%$ in group A, $\mathrm{B}, \mathrm{C}$ and $\mathrm{D}$, respectively. A marked difference was observed between groups $\mathrm{D}$ and $\mathrm{C}$ and groups $\mathrm{A}$ and $\mathrm{B}(\mathrm{P}<0.05)$. There 
$\mathbf{A}$
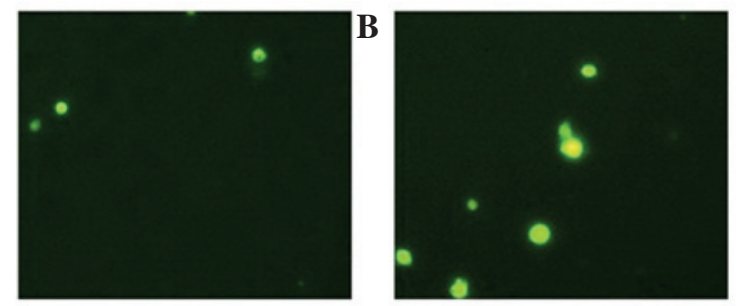

C
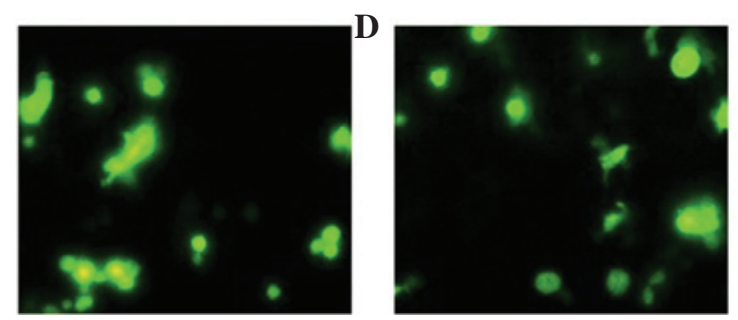

Figure 1. Fluorescence microscopy images of apoptotic cancer cells (magnification, x300). (A) Group A (control group); (B) group B; (C) group C; (D) group D.

was no significant difference between the number of apoptotic cells between groups A and B ( $>0.05)$ (Fig. 1).

\section{Discussion}

Gene therapy has been an effective strategy in the treatment of cancer. The ultrasound cavitation effect may mediate gene transfection, which could be enhanced by microbubble contrast agent pretreatment $(5,6)$. Our previous study (7) demonstrated that ultrasound treatment in the presence of an ultrasound contrast agent may significantly increase the killing of vascular endothelial and hepatic cancer cells by the HSV-TK/GCV and $\mathrm{CD} / 5-\mathrm{FC}$ system. We also revealed that ultrasound treatment is able to increase vascular endothelial permeability in mice with subcutaneously transplanted hepatic cancer and increase the efficiency of TK gene transfection.

In the present study, KDR-TK, AFP-TK and a microbubble contrast reagent were intratumorally injected into nude mice. Ultrasound treatment was conducted for 3 consecutive days and mice were administered with two prodrugs (GCV and 5-FC). This study aimed to investigate the therapeutic effect of HSV-TK/GCV and CD/5-FC on hepatic cancer in vivo. The HSV-TK gene encodes TK which may convert inactive GCV into diphosphorylated GCV. The latter may be converted into toxic triphosphorylated GCV in the presence of intracellular enzymes. The triphosphorylated GCV is able to significantly inhibit DNA polymerase and, under the regulation of the KDR gene promoter, specifically damage the vascular endothelial cells in the tumor. $\mathrm{CD}$ is able to convert inactive 5-FC into highly toxic chemotherapeutic $5-\mathrm{FU}$. This may specifically inhibit thymidylate synthetase in hepatic cancer cells under regulation by the AFP gene promoter and inhibit the synthesis of DNA, RNA and proteins, resulting in cell death. Our results demonstrated that the tumor volume in the treatment groups was comparable to that in the control group, but the tumor growth inhibition rate in the treatment groups was markedly higher than that in the control group $(\mathrm{P}<0.05)$. We also revealed that there was no marked difference in the survival time between any two groups, and the number of apoptotic cells in the treat- ment groups was significantly higher than that in the control group $(\mathrm{P}<0.05)$. Additionally, we identified that, except at the needle track, necrosis was not observed in any tumor. These findings demonstrated that the treatment in this study was not able to completely remove the hepatic cancer. This treatment has no significant control of tumor growth, and has no influence on the survival time of cancer-bearing mice; however, it may increase the tumor inhibition rate, which may be attributed to the increase in the number of apoptotic cancer cells $(8,9)$.

Our results demonstrate that ultrasound treatment in the presence of a microbubble contrast agent is an effective method mediating gene transfection. The contrast agent serves as a carrier with target genes, which reach the blood vessels at the target sites via the circulation. Following ultrasound treatment, the genes are released and gene transfection is enhanced by the cavitation effect of ultrasound (10). However, currently, the genes and microbubbles are largely injected via the tail vein, which often produces unsatisfactory efficacy. This may be attributed to the small amount of genes and microbubbles. Intratumoral injection also has the disadvantage of an uneven distribution. These may be the causes of the varying results obtained between in vitro and in vivo studies. In addition, our results revealed that there was no marked difference in the tumor growth inhibition rate between the ultrasound treatment group and the microbubble and ultrasound treatment group, which may be correlated with the short observation time or the small cohort number.

Microbubble injection in combination with ultrasound may serve as a new strategy for gene therapy as it has been demonstrated to inhibit the tumor growth rate. However, further studies are required to investigate the role of microbubble and ultrasound treatment in the gene therapy of hepatic cancer.

\section{References}

1. Degrève B, De Clercq E and Balzarini J: Bystander effect of purine nucleoside analogues in HSV-tk suicide gene therapy is superior to that of pyrimidine nucleoside analogues. Gene Ther 6: 162-170, 1999.

2. Zarnitsyn VG and Prausnitz MR: Physical parameters influencing optimization of ultrasound-mediated DNA transfection. Ultrasound Med Biol 30: 527-538, 2004.

3. Dijkmans PA, Juffermans LJ, Musters RJ, et al: Microbubbles and ultrasound: from diagnosis to therapy. Eur J Echocardiogr 5: 245-256, 2004.

4. Ferrara K, Pollard R and Borden M: Ultrasound microbubble contrast agents: fundamentals and application to gene and drug delivery. Annu Rev Biomed Eng 9: 415-447, 2007.

5. Aoi A, Watanabe Y,Mori S, et al: Herpes simplex virus thymidine kinase-mediated suicide gene therapy using nano/microbubbles and ultrasound. Ultrasound Med Biol 34: 425-434, 2008.

6. Lu QL, Liang HD, Partridge T and Blomley MJ: Microbubble ultrasound improves the efficiency of gene transduction in skeletal muscle in vivo with reduced tissue damage. Gene Ther 10: 396-405, 2003.

7. Tang Q, Xu HX, lv MD, Nie F, Yang H and Wang Y: Ultrasound contrast agent enhancing changes of murine hepatocellular carcinoma microvascular permeability caused by ultrasound irradiation. Chin J Ultra Med 22 :411-413, 2006.

8. Sheikov N, McDannold N, Sharma S and Hynynen K: Effect of focused ultrasound applied with an ultrasound contrast agent on the tight junctional integrity of the brain microvascular endothelium. Ultrasound Med Biol 34: 1093-1104, 2008.

9. Schratzberger P, Krainin JG, Schratzbger G, et al: Transcutaneous ultrasound augments naked DNA transfection of skeletal muscle. Mol Ther 6: 576-583, 2002.

10. LiXH,Zhou P,Wang LH, et al: The targeted gene(KDRP-CD/TK) therapy of breast cancer mediated by SonoVue and ultrasound irradiation in vitro. Ultrasonics 52: 186-191, 2012. 